\title{
Prospective applications of new technologies and artificial intelligence for systematizing the results of intellectual activity
}

\author{
$L$ Novoselova ${ }^{1, *}$, and $E$ Grin $^{1}$ \\ ${ }^{1}$ Kutafin Moscow State Law University (MSAL), 9, Sadovaya-Kudrinskaya Street, Moscow, 125993, \\ Russia
}

\begin{abstract}
The article addresses the prospects of using distributed ledger technologies - blockchain and artificial intelligence - for the purpose of systematizing the rights to the results of intellectual activity for their subsequent commercialization. The authors describe the key characteristics of the distributed ledger technology and review various legal problems pertaining to the use of blockchain technologies. The authors draw conclusions regarding the prospects of using blockchain and artificial intelligence technologies as measures for rapid prevention and elimination of intellectual rights violations. They also express their views on the process of commercializing intellectual property and reducing the number of conflicts related to the inclusion of intellectual property objects into distributed ledger systems. The article was prepared with the financial support of the Ministry of Higher Education and Science of the Russian Federation within the framework of the research "Scientific and methodological support for the development of theoretical and applied legal structures (models) of accounting and disposal of rights to the results of intellectual activity (technology transfer)
\end{abstract}

\section{Definition and key characteristics of the distributed ledger technology that are relevant for the determination of the prospects of its use}

The rapid development and transformation of the digital environment gives rise to new opportunities to construct registration systems for intellectual property rights. Centralized government registers (record-keeping systems) in their current state cannot ensure reliable or efficient turnover in intellectual rights.

Blockchain technologies based on distributed ledgers are now one of the most effective solutions for registration systems for intellectual property rights in the digital sphere.

The primary advantage of a distributed ledger is the lack of central authority in the management of the database, which makes this mechanism the most suitable for exclusive rights registration systems. The distributed ledger database is spread across several nodes (devices) on a peer-to-peer network, where each replicates and saves an identical copy of the

\footnotetext{
*Corresponding author: la-novosielova@ yandex.ru
} 
ledger and updates itself independently. When a ledger update happens, each node constructs the new transaction, and then the nodes vote by consensus algorithm on which copy is correct. Once a consensus has been determined, all the other nodes update themselves with the new, correct copy of the ledger.

In the past ten years, one of the distributed ledger technologies - blockchain - has become increasingly popular.

Technically speaking, blockchain is a technology for organizing databases that rely on the Internet and uses its advantages, including its open protocol and the ability to make calculations and encrypt information. This distributed database of transactions can be compared to an accounting ledger where each new transaction is recorded immediately after a previous transaction and where it is impossible to modify or destroy previous records. This ledger is active, compiled in chronological order, distributed, verifiable and protected from any falsifications by the system of distributed trust (consensus) between the participants in the system (nodes).

The system alone cannot guarantee that data is reliable, because its data is entered into the system by users. In addition, the data entered in the system may, in time, deviate from real facts.

For example, let's describe the system of bitcoin blockchain functioning. It may be divided into four stages:

1) the participants agree on the terms of the transaction (transfer of money or other assets);

2) the system of transaction records is verified by each member of the network. By analyzing the chronology of records, it can be verified that the person actually possesses the assets he is trying to dispose of.

3 ) if the results of verification are positive, the transaction is confirmed, and the last block is added to the system;

4) the system of records is distributed to all the participants in the network, which guarantees its safety, because each person has to change his record in order it to be changed in the system.

Currently, cryptography is used for: 1) signing the transaction; 2) signing the block.

This ensures 1) verification of the transaction and the block, 2) verification of the authority of the person who created the transaction or the block, and, consequently 3) eliminates the possibility of several transfers of one and the same asset by the same person (copying of electronic value). The system only "recognizes" the encryption key.

"Blockchain uses digital signatures for authentication and ensuring integrity of transactions (and sometimes blocks). In the case of cryptocurrency, the process of authentication means that money can only be spent by the person who received it in a previous transaction. Accordingly, there is no risk of "double spending" (disposal), if we speak of the systems that are used to transfer (provide) intellectual rights. The special nature of blockchain is in the fact that the information on authentication is built into each transaction and not separated from the business logic - this is why blockchain is considered to be more secure. In ordinary systems, the authentication mechanism can be hacked or administratively avoided and the backend can be manipulated. In blockchain, by definition, it cannot be done" [1].

Therefore, the specific features of blockchain technologies are as follows:

a) the fixation of asset ownership or actions performed is done without an intermediary, but directly by the participant (participants) in the system;

b) there is no centralized storage of information - it is distributed among the computer systems of the participants who have direct control over the entire data content. 
In blockchain-based systems, decentralization means not only that data is stored in a distributed system, but also that different mechanisms are used for the making and verification of transactions where there is no single intermediary responsible for the accuracy of records.

Classic asset inventory systems are based on trust in a particular intermediary (for example, a bank in case of payment and settlement systems, or a registrar in case of securities registration, or the state in the system of record keeping in relation to property or exclusive rights). All current rules are designed around this system.

The absence of a single intermediary changes the character of regulation in a fundamental way, because there is no specific person responsible for the accuracy of records or who can modify records, provide information, etc.

The spread of blockchain technologies has called for a need to regulate the new relationships that they have given rise to. Blockchain is used in various areas (government, healthcare, insurance, energy, delivery systems, payments and settlements, etc.). Its potential has not been fully exploited yet. The appearance of new technologies with a wider range of functions and higher speeds and lower risk performance is also expected.

Blockchain systems can be controlled (existence of one or several subjects with special rights who have special authority) and non-controlled (equal rights, no subjects with special rights or authority).

In order to describe the processes within a blockchain system the term "token" is used (synonyms: "coin", “crypto-token", “cryptocurrency"). It stands for a digital marker or a means of access, or a mechanism for tracking certain activities.

Depending on the purpose of the technology, a token can be a marker of identity, action, or a digital image of almost any kind of property, etc.

When the technology is used to transfer (provide) a subject of transaction (a separate unit of value), such transfer is performed in the blockchain by connecting it to a specific block of information in this system (a token).

For this, tangible and intangible assets must be "transferred" into the virtual environment and reflected in virtual form.

Both intangible (uncertificated securities, undocumented rights of claim, etc.) and tangible assets (property, commodities) can be tokenized.

The real novelty of blockchain, however, is not only in the fact that a distributed system of data storage is used or in the security system applied, but because it creates the possibility of setting transaction rules (the business logic) in relation to each specific transaction. This makes blockchain different from common databases, where the rules are often established at the level of the entire database or application, but not for a single transaction.

Blockchain makes it possible to execute "smart contracts", i.e. contracts that can be partially or fully executed or enforced without human interaction [2].

Another specific feature of blockchain is the ability to set time tags (stamps). In essence, the entire network checks the status of certain data (hash) at a particular time. Accordingly, a decentralized network makes it possible to confirm the presence of something (event, fact, information) at a particular time - such confirmation can be used as evidence in court. Before now, this could only be done by centralized services or notaries.

In the context of protection and confirmation of exclusive rights (title) to an object of digital property, the mechanism of a distributed ledger (in particular, blockchain) can be considered as one of the ways to build a system of registration of author's rights (copyright). Blockchain makes it possible to improve the protection of the holder of the title to digital objects thanks to the principles of distribution and engagement of a large number of 
participants in the system. The system also makes it possible to certify documents. For example, software developers can create unique hashes for each version of the code; inventors can prove that they got a certain idea at a particular time; and authors can protect their works using the data in the register as evidence.

The introduction of distributed ledgers based on blockchain cannot fully eliminate violations in intellectual rights or copyright disputes; however, it can provide title holders with a reliable evidence base in case of any copyright disputes, which is why blockchain can be considered a system that is not just capable of supplementing the current systems of intellectual property management, but of fully replacing them.

In Russia, one of the most widely-known intellectual property registers created on the basis of blockchain is IPCHAIN.

IPCHAIN, as defined by its developer (National Coordination Center for Processing Transactions with Rights and Objects of Intellectual Property) - is a blockchain based digital trust-space: a decentralized distributed registry of intellectual property that makes it possible to keep the information on the objects of intellectual property stored in this register unchanged.

In reality, IPCHAIN is not just a ledger, but an infrastructural mechanism designed to record the rights to the results of intellectual activity and to facilitate interaction between persons in relation to the rights to objects of intellectual property, the information then being stored in the register. In addition, in the IPCHAIN mechanism, a certain role is assigned to an "inspector" - a person who searches for the rights to objects of intellectual property and provides statistics on the transaction ledger. In addition to the registry's identity check function during the registration of new objects, the "inspector's" function can increase the level of transaction security and convenience.

Various services for the registration and/or use of objects of intellectual property can be created on the basis of IPCHAIN. For example, FONMIX is a service for legal background sound, video branding and sound design used by various facilities (from restaurants and hotels to shopping centers and offices); DR\&D Network - is a service for organizing public digital research institutes, which aims to develop, implement and manage the rights to the results of applied science projects; Online Patent - is a digital service for patenting in Russia and abroad; FAN (Free Analytic Network) — is a service for depositing, legal protection, and circulation of databases storing initial social and economic information, as well as methods and technical solutions for their processing and legal distribution of analytical information; I3D - is a service for depositing, managing, and assessing 3D service utility models and detecting their confusing similarity with the help of neuro networks.; IP VALUE is a service for assessing the economic potential of IP objects use; IPEX is a designer platform for all types of transactions with IP rights and objects, including cross-border agreements; $\mathrm{CO}-\mathrm{FI}$ - a digital crowd-funding platform providing funding for creative and technological projects, including investment loans secured by intellectual property; DI'S PUT is a platform for alternative resolution of disputes related to the IP sphere, which brings together arbitrators, experts and disputing parties.

Accordingly, the capabilities of the national decentralized blockchain-based registry IPICHAIN are currently determined by the minimization of the role of intermediaries in the interaction of consumers and producers of objects of intellectual property; the guarantee of invariability and absence of false "life stories" of IP objects; the ability to store, verify and exchange information on objects of intellectual property; infrastructural capabilities to create digital services using verified information on objects of intellectual property and the rights to them. 


\section{Ways of using blockchain technologies for registration and systematization of intellectual rights}

A large number of projects based on blockchain are being implemented in the system of IP creation, registration and turnover. The technical solutions available today can help to create and operate systems for the following:

1) depositing author's rights (copyright);

2) raising investment to finance the development of a creative result;

3) engaging and coordinating specialists (crowd-sourcing) to create the desired result;

4) commercializing protected results of intellectual activity (intellectual property exchanges, services that provide paid access to products, objects of related rights, etc.);

5) distribution of revenue from the use of the results of intellectual activity (IA) and brands among legal owners;

6) ensuring free legal access to the results of IA;

7) identifying and blocking plagiarism;

8) filing applications for the provision of legal protection to the results of IA, managing such applications (withdrawal, amendment, etc.), tracking the status of such applications with the relevant authorities;

9) settling disputes, including those related to the determination of authorship or the fact of breach of copyright, in an alternative procedure for dispute resolution (similar to arbitration).

This list of areas related to the creation, use and protection of intellectual property where a positive effect of blockchain technologies can be observed is not exhaustive; indeed, the technologies offered by developers can do far more than efficiently deal with the procedures and challenges specifically listed above.

Various projects based on the use of blockchain technologies are most widely used in the modern music industry [3].

Thus, by using the service Bittunes, users can gain direct access to the creations of independent artists and pay for using such creations with cryptocurrency.

The blockchain startup Stem raised financing in the amount of $\$ 4.5$ million, and its users got the opportunity to upload their music and video to YouTube, Spotify, Apple Music and Soundcloud, were able to track the use of their creations and distribute their revenue in a fair way in accordance with the relevant programmed smart contracts. The platform Blokur currently uses a solution that excludes intermediaries and ensures that the artist is paid directly. The startup Smilo has created a trading platform for music fans. The pricing model is based on an algorithm that uses the supply and demand variable. The price of content increases with every unit sold [4].

Blockchain technology has long been used in the music industry to record information on copyright and related rights to music and music performance. It has also been employed for the distribution of royalties and various types of investment procedures [5].

The technology is equipped to search for and promote new artists. Thus, it says on the main page of the PeerTracks website that it is "the best universal music platform" both for music fans and for artists. The system attaches a "smart contract" to each song that is uploaded by an artist, and the contract automatically distributes the revenue depending on the transaction made between the artist, the author of the song, the composer and other members of the band. Artists get their own tokens that bear their names and are similar to virtual baseball cards. The artist sets the number of available tokens, so there may be a limited number of them. The concept is simple - they create a so-called store of valuable things whose price depends on the artist's popularity. 
Users get a free full on-demand access to the entire music catalogue of PeerTracks. Moreover, the music is not interrupted by commercials. Users can save songs and playlists to be used offline and download any tracks or albums from the catalogue. Users can also buy artists' tokens and resell them. As the artist's popularity grows, the value of their tokens also increases; accordingly, users can make a profit if they started supporting an artist before he or she became popular. If you buy his/her tokens, you will enjoy exclusive privileges - you will get various gifts and benefits from the artist. This motivates people to become active promoters, while the artist gets a community of active fans who are ready for long-term collaboration. The creators of the system are certain that talent scouts will definitely notice new tracks or songs, because the users of the platform make them noticeable [6].

Blockchain technologies are used for other objects such as photography (Mediachain start-up) [7] and artistic images of limited circulation (Swaincoin) [8]. Artlery employs bitcoin blockchain in several ways. The system registers the origin of a work of art as metadata in blockchain using its partnership and integration with another startup - Ascribe.io. The system uploads the payment spreadsheet in such a way that all participants are immediately paid their portion of the revenue depending on their shares. Initially, the platform was oriented at visual arts, but it has a good chance for success in other areas related to copyright, such as music, books and film [9].

\subsection{Verification of the rights of authors and proprietors}

The existing systems for the registration of copyright and related rights based on blockchain have one negative characteristic in common - their data on authors and proprietors is not publicly reliable(??) while the records in such registers, in and of themselves, do not confirm that the persons listed in the register have particular exclusive rights.

The possibility of alleviating the problem using technical means (preliminary records monitoring, information in social networks) does not eliminate the need to establish legal mechanisms that minimize the risks incurred by users of such systems.

In particular, such users bear the risk of liability for illegal use of exclusive rights when purchasing such rights from an unauthorized person.

Due to the fact that many systems are oriented not just at the end-consumer, but at ensuring the circulation of exclusive rights (execution of transactions for the disposal of exclusive rights resulting in a change of proprietors), the buyers of such rights also run significant risks considering the fact that, for example, they are not subject to bad-faith defense.

In addition to standard solutions to the problem, such as the introduction of stricter sanctions against persons who entered inaccurate information in the register, other alternative methods are also required.

Admittance to the market of technical services for registration and commercialization of intellectual rights must be regulated. For legal admittance to such a market, service providers must provide information on compliance with the relevant minimum standards of the technical services used for verification of authorship (proprietorship); they must do the same with regards to insurance of the risks of system users at the expense of the persons that provide blockchain services, or of a fund created by persons whose rights are registered in the system. At the same time, the requirements of compliance with particular standards for the purpose of admission to the market must be predominantly oriented at services that are created by third parties. As far as authors are concerned, their access to the market must be made as free as possible. 
Experts also note that "in order to maximize the power of distributed ledgers, these may need to be interoperable with other ledgers. However, maximizing the potential of interoperability goes far beyond interoperability of authentication - it requires agreements about data interoperability, policy interoperability and the effective implementation of international standards" [10].

As a guarantee of the existence of a person's authorship, (copyright) rights existing registry systems currently use the mechanism of moderation - confirmation of an indicated fact by an independent expert community. The mechanism does not provide a binary solution - whether or not the indicated right exists, but information on the degree of likelihood. For example, none of the experts confirms authorship, or authorship is confirmed by 5 experts, or authorship is confirmed by more than 100 experts. The systems also use the ratings of the experts by determining the average weight of the score of a specific expert. Incorrect or baseless scores reduce the weight - significance of the expert's score.

Obviously, such mechanisms do not take away the author's right to prove his/her authorship in a different way, including in a court of law, or to challenge the authorship of persons who provided false information to the registry. In relation to the mechanisms of confirmation of the authenticity of the information on authorship described above, a possibility of accepting this data as evidence must be provided, and liability of persons providing intentionally false opinions on authorship must be established.

If the system is built on blockchain, no special guarantee of safekeeping and invariability of data is required. However, this does not exclude the need for adjustment or preservation of the accuracy of records entered in the data ledger. At the stage when real information is transferred into the digital environment, there is always a risk of error or misrepresentation. The point of entry must always be the point of control in any system [11].

Accordingly, the requirements to registration systems must incorporate requirements for ledger adjustment and verification of the data entered into it.

Artificial intelligence technologies combined with blockchain technologies are also capable of producing a positive effect, in particular, for the purpose of identifying object with analogous content, identification of instances of illegal use and generation of flexible terms of execution in "smart contracts".

\subsection{The problem of protecting the same result of intellectual activity using different constructs}

In cases of exclusive rights, the problem where one and the same result of intellectual activity can be protected using different legal models is also valid. Thus, the form of an item may be protected as an object of copyright, as a utility model and as a trademark. The relationship of rights to such "parallel objects" creates certain legal difficulties, and when the process of their disposal is activated, this can give rise to serious legal risks.

More problems appear when separate parts of protected works (characters, names, quotes) are circulated independently.

This may result in various collisions of the rights of purchasers of such objects, the rights to which were purchased in different blockchain systems. The algorithm of the solution is based on the approach described previously. Priority, for example, may be granted to the person who acquired an exclusive right for the entire object, or executed a licensing agreement for the widest range of rights.

Legislative solutions concerning the matter of priority in cases of transfer of rights to the entire work and of rights to a protected portion of the work through various systems may be 
different, but in any case, the proprietor must bear the responsibility of reporting the facts of inclusion of related objects in other registries. This in turn must be supported by strict property sanctions. Blockchain technologies, including those used in combination with artificial intelligence, are capable of providing a solution to a wide range of tasks related to the creation of active intellectual property registry systems.

Active intellectual property registry systems should be understood as systems that are not only used to register the results of intellectual activity and title to IP objects, but that are also directly used to execute contracts for the use of such objects, register encumbrances and reflect any changes in proprietorship. The use of the indicated technologies can fundamentally change the architectonics of the intellectual property registry systems and to significantly affect their functions. In addition to thorough technical testing of various vulnerabilities and systems stability, an essential condition for the development of blockchain-based registries in Russia would be the ability to work with other systems.

Legal mechanisms play an important role in the successful implementation of the possibilities built into various technologies. On the one hand, the existing unjustified legal obstacles for using the relevant technologies need to be eliminated. On the other hand, logical regulation that helps to open up the useful potential of technologies is required. We are not speaking only of government regulation - self-regulation systems and alternative conflict prevention and resolution mechanisms need to be developed and supported as well.

The wide use of blockchain and artificial intelligence technologies for IP commercialization purposes and the increasing number of conflicts related to the inclusion of IP objects in various registries based on these technologies require prompt development and implementation of measures designed to reduce the legal risks that arise.

\section{References}

1. Testova A Cryptography in blockchains: hash functions, keys and digital signatures https: habrahabr.ru/company/bitfury/blog/327272/

2. Saveliyev A 2016 Electronic commerce in Russia and abroad: legal regulation (M.: Statut SPS “KonsultantPlus”)

3. 2016 Music On The Blokchain. Blockchain For Creative Industries Research Cluster. Middlesex University Report (London: Middlesex University)

4. 10 projects on the blockchain in the music industry https://bits.media/news/blokcheynkak-revolyutsiya-v-muzykalnoy-industrii/

5. Walach D A 2014 Bitcoin for Rockstars. How Cryptocurrency Can Revolunionize the Music Industry https://www.wired.com/2014/12/ bitcoin-for-rockstars/

6. 2015 PeerTracks Inc http.peertracks.com

7. Mediachain http://www.mediachain.io

8. Swincoin http://swincoin.com

9. Tapscott D 2017 Blockchain revolution: How the technology behind Bitcoin and other cryptocurrencies is changing the world today (M.: Exmo) p 313

10. A report by the UK Government Chief Scientific Adviser «Distributed Ledger Technology: beyond block chain» https://bit.ly/1WreVPL

11. Gyr'yeva M 2016 Information and analytical edition about matters of infrastructure of the securities market in Russia and the CIS 412 